\title{
Brachytherapy with Intratumoral Injections of Radiometal- Labeled Polymers That Thermoresponsively Self-Aggregate in Tumor Tissues
}

\author{
Kohei Sano*1-3, Yuko Kanada*1, Kengo Kanazaki ${ }^{1,4}$, Ning Ding ${ }^{1}$, Masahiro Ono ${ }^{1}$, and Hideo Saji ${ }^{1}$ \\ ${ }^{I}$ Department of Patho-Functional Bioanalysis, Graduate School of Pharmaceutical Sciences, Kyoto University, Kyoto, Japan; ${ }^{2}$ Kyoto \\ University Hospital, Kyoto, Japan; ${ }^{3}$ Department of Biophysical Chemistry, Kobe Pharmaceutical University, Kobe, Japan; and \\ ${ }^{4}$ Medical Imaging Project, Corporate R\&D Headquarters, Canon Inc., Tokyo, Japan
}

Brachytherapy is a type of radiotherapy wherein titanium capsules containing therapeutic radioisotopes are implanted within tumor tissues, enabling high-dose radioirradiation to tumor tissues around the seeds. Although marked therapeutic effects have been demonstrated, brachytherapy needs a complicated implantation technique under general anesthesia and the seeds could migrate to other organs. The aim of this study was to establish a novel brachytherapy using biocompatible, injectable thermoresponsive polymers (polyoxazoline [POZ]) labeled with ${ }^{90} \mathrm{Y}$, which can self-aggregate above a specific transition temperature $(\mathrm{Tt})$, resulting in long-term intratumoral retention of radioactivity and therapeutic effect. Therefore, we evaluated the tumor retention of radiolabeled POZ derivatives and their therapeutic effects. Methods: Using oxazoline derivatives with ethyl (Et), isopropyl (Isp), and propyl (Pr) side chains, we synthesized EtPOZ, IspPOZ, Isp-PrPOZ (heteropolymer), and PrPOZ and measured their characteristic Tts. The intratumoral retention of ${ }^{111} \mathrm{In}$-labeled POZ was evaluated until $7 \mathrm{~d}$ after injection in nude mice bearing PC-3 human prostate cancer. The intratumoral localization of ${ }^{111} \mathrm{In}$-labeled POZ derivatives was investigated by an autoradiographic study. Furthermore, a therapeutic study using 90Y-labeled Isp-PrPOZ was performed, and tumor growth and survival rate were evaluated. Results: The Tts of EtPOZ, IspPOZ, Isp-PrPOZ, and PrPOZ $(\sim 20 \mathrm{kDa})$ were greater than $70^{\circ} \mathrm{C}, 34^{\circ} \mathrm{C}, 25^{\circ} \mathrm{C}$, and $19^{\circ} \mathrm{C}$, respectively. In the intratumoral injection study, Isp-PrPOZ and PrPOZ $(2,000 \mu \mathrm{M})$ with Tts lower than tumor temperature $\left(33.5^{\circ} \mathrm{C}\right.$ under anesthesia) showed a significantly higher retention of radioactivity at $1 \mathrm{~d}$ after injection (73.6\% and $73.9 \%$, respectively) than EtPOZ (5.6\%) and IspPOZ $(15.8 \%)$. Even at low injected dose $(100 \mu \mathrm{M})$, Isp-PrPOZ exhibited high retention $(68.3 \%$ at $1 \mathrm{~d})$. The high level of radioactivity of Isp-PrPOZ was retained in the tumor $7 \mathrm{~d}$ after injection (69.5\%). The autoradiographic study demonstrated that the radioactivity of ${ }^{111}$ In-labeled Isp-PrPOZ and PrPOZ was localized in a small area. In the therapeutic study using ${ }^{90}$ Y-labeled Isp-PrPOZ, significant suppression of tumor growth and prolonged survival rate were achieved in an injection dose-dependent manner compared with that observed for the vehicle-injected group and nonradioactive IspPrPOZ-injected group. Conclusion: The injectable ${ }^{90}$ Y-labeled Isp-PrPOZ was retained for a prolonged period within tumor tissues

Received Jan. 16, 2017; revision accepted Apr. 3, 2017.

For correspondence or reprints contact: Kohei Sano, Department of Biophysical Chemistry, Kobe Pharmaceutical University, 4-19-1 Motoyama Kitamachi, Higashinada-ku, Kobe, Japan, 658-8558.

E-mail: ksano@kobepharma-u.ac.jp

${ }^{*}$ Contributed equally to this work.

Published online Apr. 13, 2017.

COPYRIGHT (C) 2017 by the Society of Nuclear Medicine and Molecular Imaging. via self-aggregation and exhibited marked therapeutic effect, suggesting its usefulness for brachytherapy.

Key Words: brachytherapy; polyoxazoline; thermoresponsive polymer; radiotherapy

J Nucl Med 2017; 58:1380-1385

DOI: 10.2967/jnumed.117.189993

B rachytherapy is a form of radiotherapy with seeds (therapeutic radioisotopes) that are implanted within tumor tissues. These tissues can then receive high-dose radioirradiation. Compared with chemotherapy, hormone therapy, and external-beam radiation, brachytherapy can provide a long-term antitumor effect with decreased damage to normal tissues; marked therapeutic effects have been demonstrated in particular to prostate, breast, and brain cancers $(1,2)$. The global market for brachytherapy is expected to reach over U.S. $\$ 2.4$ billion in 2030 . However, brachytherapy requires a complicated implantation technique under general anesthesia. Seed migration is also a well-recognized phenomenon after implantation. Furthermore, seed removal can be required, although rarely (3). Therefore, the development of injectable radiopharmaceuticals that are rigidly retained in the tumor tissues, instead of capsule-type seeds, is desired to allow for more simple and harmless procedures (4-7).

Poly (2-alkyl-2-oxazoline) (POZ), an isomeric polypeptide material that can be synthesized via living cationic ring-opening polymerization (8), is garnering attention as a biocompatible and hydrophilic biomaterial for drug delivery $(9,10)$, in addition to the more traditional polyethylene glycol. Recently, we have demonstrated that chemically modified POZ accumulates in tumor tissue via an enhanced permeability and retention effect and is rapidly cleared from the blood after intravenous injection of the probe (11), indicating its usefulness for biomedical applications. Moreover, POZ has a lower critical solution temperature and can self-aggregate above a specific transition temperature $(\mathrm{Tt})$ that is dependent on the oxazoline composition and molecular weight of its polymers $(12,13)$, which can be controlled in a range of $10^{\circ} \mathrm{C}-90^{\circ} \mathrm{C}$. This thermoresponsive characteristic of POZ motivated us to develop an injectable POZ radiopharmaceutical suitable for brachytherapy; ultimately, we designed therapeutic, radiolabeled POZ derivatives that are soluble at room temperature (RT) but rapidly self-aggregate and are rigidly retained in the tumor on intratumoral injection (Fig. 1). 


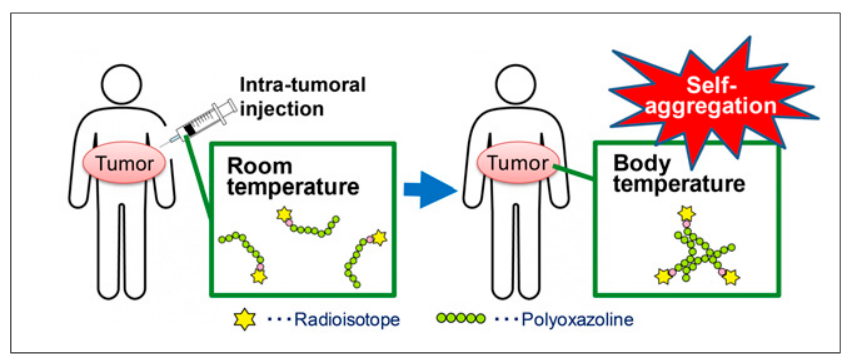

FIGURE 1. Concept of injectable polymer (POZ) labeled with radiometals that are thermoresponsively self-aggregated in tumor tissues for brachytherapy.

In the present study, we synthesized POZ derivatives with different Tts and determined their physicochemical properties. A metal chelator, DOTA derivative $(14,15)$, was then introduced to the POZ derivatives before labeling with either ${ }^{111} \mathrm{In}$ ( $\gamma$-ray-emitting radiometal, 245 and $171 \mathrm{keV}$; half-life, $67.9 \mathrm{~h}$ ) or ${ }^{90} \mathrm{Y}$ ( $\beta^{-}$-rayemitting radiometal, $2.28 \mathrm{MeV}$; half-life, $64.0 \mathrm{~h}$ ). We first evaluated the tumor retention rate of ${ }^{111}$ In-labeled $\mathrm{POZ}$ derivatives to estimate their therapeutic effect using prostate cancer-implanted mice. We also performed a therapeutic study of ${ }^{90} \mathrm{Y}$-labeled $\mathrm{POZ}$ derivatives and evaluated whether POZ-based biocompatible radiopharmaceuticals could provide long-term tumor retention and antitumor effect.

\section{MATERIALS AND METHODS}

\section{Reagents}

Methyl $p$-toluenesulfonate, ethylenediamine, and super-dehydrated acetonitrile were purchased from Wako Pure Chemical Industries, Ltd. 2-ethyl-2-oxazoline, 2-isopropyl-2-oxazoline, and 2-propyl-2oxazoline were purchased from Tokyo Chemical Industry Co., Ltd. S-2(4-isothiocyanatobenzyl)-1,4,7,10-tetraazacyclododecane tetraacetic acid ( $p$-SCN-Bn-DOTA) was purchased from Macrocyclics Inc. ${ }^{111} \mathrm{In}$ chloride $\left({ }^{111} \mathrm{InCl}_{3}\right)$ was kindly supplied by Nihon Medi-Physics. ${ }^{90} \mathrm{Y}$-chloride $\left({ }^{90} \mathrm{YCl}_{3}\right)$ was purchased from Eckert \& Zieglr Radiopharma GmbH. Other reagents were of reagent grade and were used without further purification unless otherwise indicated.

\section{Synthesis of Amine-Terminated POZ Derivatives}

Thermoresponsive POZ derivatives including EtPOZ composed of 2-ethyl-2-oxazoline, IspPOZ composed of 2-isopropyl-2-oxazoline, IspPrPOZ composed of 2-isopropyl-2-oxazoline and 2-propyl-2-oxazoline, and PrPOZ composed of 2-propyl-2-oxazoline were synthesized according to previous reports $(11,16)$. The reaction scheme is shown in Figure 2. To prepare EtPOZ, IspPOZ, Isp-PrPOZ, and PrPOZ, methyl $p$-toluene sulphonate (1 eq) was mixed with 2-ethyl-2-oxazoline (400 eq), 2-isopropyl-2-oxazoline (500 eq), a mixture of 2-isopropyl-2-oxazoline (200 eq) and 2-propyl-2-oxazoline (300 eq), or 2-propyl-2-oxazoline (500 eq) in acetonitrile, respectively, and then stirred for $14 \mathrm{~min}$ at $140^{\circ} \mathrm{C}$ under microwave irradiation (Discover; CEM Co.). Molecular weight was determined by gel permeation chromatography with a KD804 column (Showa Denko) using polyethylene glycol as a calibration standard. Molecular weights were 19,712, 18,204, 17,757, and 18,235 for EtPOZ, IspPOZ, Isp-PrPOZ, and PrPOZ, respectively.

Subsequently, ethylenediamine (EDA) was added to the reaction solution and stirred for an additional $7 \mathrm{~min}$ at $140^{\circ} \mathrm{C}$ under microwave irradiation. After the reaction, the mixture was cooled to RT and the solvent was evaporated. The mixture was dissolved in methanol and dialyzed against methanol with pretreated regenerated cellulose membrane Spectra/Por 7 dialysis tubing (molecular weight cutoff, $3.5 \mathrm{kDa}$; Spectrum Laboratories, Inc.). Each amino-terminated POZ derivative is hereinafter referred to as EtPOZ-EDA, IspPOZ-EDA, Isp-PrPOZ-EDA, or PrPOZ-EDA, according to the composition of oxazoline. Molecular weights were 19,898, 17,892, 17,494, and 17,676 for EtPOZ-EDA, IspPOZ-EDA, Isp-PrPOZ-EDA, and PrPOZ-EDA, respectively.

\section{Measurement of Phase Tt}

POZ derivatives were dissolved in phosphate-buffered saline (PBS) $(2,000 \mu \mathrm{M})$, and their Tts were measured by a Zetasizer Nano (Malvern Instruments Ltd.) device with heating at a rate of $1^{\circ} \mathrm{C} / \mathrm{min}$. To measure phase transition time, a solution of EtPOZ or Isp-PrPOZ $(2,000 \mu \mathrm{M}, 5 \mu \mathrm{L})$ was dropped onto hot plate heated at $36.5^{\circ} \mathrm{C}$ (close to body temperature).

\section{Preparation of ${ }^{111}$ In-Labeled POZ Derivatives}

For radiolabeling with ${ }^{111} \mathrm{In}$ or ${ }^{90} \mathrm{Y}, \mathrm{POZ}-\mathrm{EDA}$ derivatives prepared as mentioned above were conjugated with $p$-SCN-Bn-DOTA, a metal chelator (Fig. 2). Each POZ-EDA derivative was reacted with $p$-SCNBn-DOTA (5-10 eq) in methanol with $\mathrm{K}_{2} \mathrm{CO}_{3}$ for $36 \mathrm{~h}$ at RT. After the reaction, unconjugated $p$-SCN-Bn-DOTA was purified by ultrafiltration with Amicon Ultra centrifugal filter units (molecular weight cutoff, $3 \mathrm{kDa}$ ) (Merck Millipore, Co.) and by gel filtration (PD-10 columns; GE Healthcare). ${ }^{111}$ In labeling of POZ-EDA-DOTA derivatives was performed by mixing and reacting with ${ }^{111} \mathrm{InCl}_{3}(9.3-13.3$ $\mathrm{MBq}$ ) in $0.1 \mathrm{M}$ acetate buffer ( $\mathrm{pH} 6.0$ ) for $10 \mathrm{~min}$ at RT. After incubation, ethylenediaminetetraacetic acid (10 eq) was added and incubated for $5 \mathrm{~min}$ at RT, and then the buffer was exchanged with PBS ( $\mathrm{pH} 7.4$ ) by ultrafiltration (Amicon Ultra-4, $3 \mathrm{kDa}$ ) to remove the unchelated ${ }^{111} \mathrm{In}$. Radiochemical purity was assessed by gel filtration (PD-10 columns) eluted with PBS.

\section{Animal Model}

Animal experiments were conducted in accordance with the institutional guidelines of Kyoto University and were approved by the Kyoto University Animal Care Committee. Human prostate cancer PC- 3 cells $\left(2 \times 10^{6}\right.$ cells suspended in the mixture of Matrigel $[50 \mu \mathrm{L}$, Corning Inc.] and $50 \mu \mathrm{L}$ of PBS) were inoculated subcutaneously into the right thigh of 5-wk-old male BALB/c-nu/nu mice (Japan SLC, Inc.). When the tumor size reached approximately $0.7-1.0 \mathrm{~cm}$ in diameter, the animals were used for in vivo experiments. The rectal and tumor temperatures were measured by thermometer or thermography.

\section{Tumor Retention Analysis}

To investigate the effect of phase Tts of POZ derivatives on tumor retention, ${ }^{111}$ In-labeled DOTA-EDA-EtPOZ ( ${ }^{111}$ In-EtPOZ), DOTA-EDAIspPOZ ( ${ }^{111}$ In-IspPOZ), DOTA-EDA-Isp-PrPOZ ( ${ }^{111}$ In-Isp-PrPOZ), and DOTA-EDA-PrPOZ ( ${ }^{111}$ In-PrPOZ) $(2,000 \mu \mathrm{M}, 37 \mathrm{kBq}, 5 \mu \mathrm{L}$ of PBS) were intratumorally injected into PC-3 tumors under isoflurane anesthesia using a nano syringe (Altair Technologies Ltd.). At 1, 3, or $7 \mathrm{~d}$ after

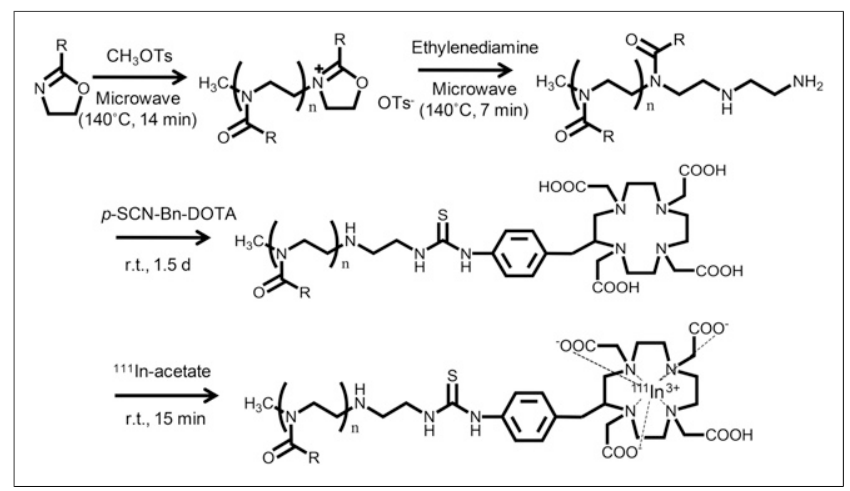

FIGURE 2. Synthesis scheme of ${ }^{111} \mathrm{In}$-labeled POZ derivatives. 
injection, the tumor was excised, and the radioactivity in the tumor was calculated using the injection dose as a standard. Data are presented as a percentage of tumor retention against $0 \mathrm{~min}$ after injection. The biodistribution of ${ }^{111} \mathrm{In}$-labeled POZ derivative to other organs was also evaluated at $1 \mathrm{~d}$ after intratumoral injection of probes. Data were calculated as percentage injected dose. Furthermore, in vivo biodistribution of probes after intravenous injection was also investigated, and the data were calculated as percentage injected dose per gram of tissue.

To evaluate the localization of ${ }^{111} \mathrm{In}$-labeled POZ derivatives in tumor tissues, the tumor tissues were excised at $1 \mathrm{~d}$ after injection. The excised tumors were frozen and cut into $20-\mu \mathrm{m}$-thick sections with a cryomicrotome. The sections were thaw-mounted in silane-coated slides, and were then placed on a phosphor image plate (BAS-SR 2040; FUJIFILM) for $1 \mathrm{~h}$ to obtain ${ }^{111}$ In autoradiograms. The autoradiographic images were analyzed using a computerized imaging analysis system (BAS2500; FUJIFILM). The same slides used in the autoradiographic study were subjected to hematoxylin and eosin staining.

To investigate the influence of molecular weight of $\mathrm{POZ}$ derivatives on tumor retention, ${ }^{111} \mathrm{In}$-Isp-PrPOZ (5 and $10 \mathrm{kDa}$, synthesized elsewhere) $(2,000 \mu \mathrm{M}, 37 \mathrm{kBq}, 5 \mu \mathrm{L}$ of PBS) was intratumorally injected into PC-3 tumors. Next, to evaluate the relation of $\mathrm{POZ}$ concentration with tumor retention, ${ }^{111} \mathrm{In}-\mathrm{Isp}-\mathrm{PrPOZ}(20 \mathrm{kDa})(100,500,2,000$, and 4,000 $\mu \mathrm{M})$ (37 kBq, $5 \mu \mathrm{L}$ of PBS) was intratumorally injected into PC-3 tumors. Tumor retention was evaluated as a percentage of tumor retention $1 \mathrm{~d}$ after injection against that 0 min after injection for each condition.

\section{Therapeutic Studies Using ${ }^{90}$ Y-Labeled Isp-PrPOZ}

For therapeutic studies using Isp-PrPOZ derivatives, Isp-PrPOZ was radiolabeled with ${ }^{90} \mathrm{Y}$ as follows. Isp-PrPOZ-EDA-DOTA (2.5 mg) was incubated with ${ }^{90} \mathrm{YCl}_{3}$ in acetate buffer (0.1 M, pH 6.0) for $90 \mathrm{~min}$ at RT. After incubation, ethylenediaminetetraacetic acid (10 eq) was added and incubated for $5 \mathrm{~min}$ at RT, and then unchelated ${ }^{90} \mathrm{Y}$ was eliminated by ultrafiltration.

Therapeutic studies were performed for 5 groups $(n=12$ for each group): PBS-injected group (control), nonradioactive yttrium-labeled

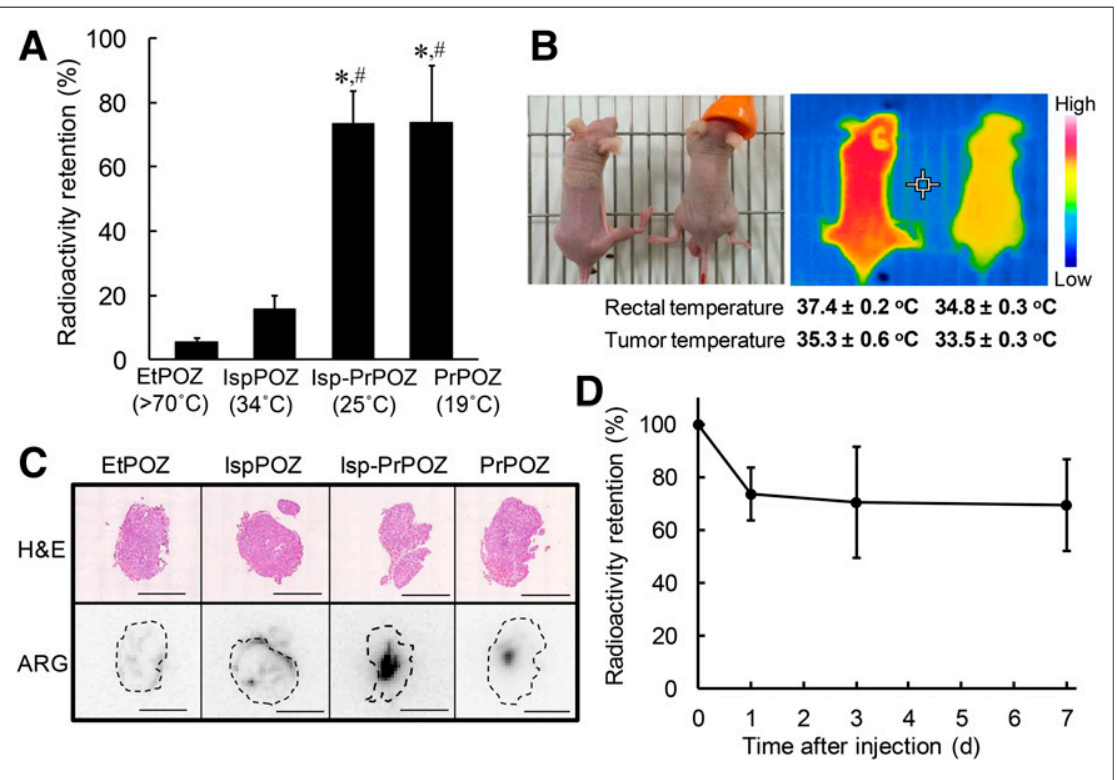

FIGURE 3. Evaluation of tumor retention of ${ }^{111} \mathrm{In}$-labeled POZ derivatives in PC-3 tumors. (A) Radioactivity retention (\%) in tumor $1 \mathrm{~d}$ after intratumoral injection of ${ }^{111} \mathrm{In}$-labeled POZ derivatives ( ${ }^{\star} P<0.01$ vs. EtPOZ and ${ }^{\#} P<0.01$ vs. IspPOZ). Data are mean \pm SD. (B) Rectal and tumor temperature with (right) or without (left) isoflurane anesthesia (2\%). (C) Localization of radioactivity in tumor determined by autoradiography. Scale bar, $10 \mathrm{~mm}$. (D) Long-term retention of radioactivity in tumor after injection of ${ }^{111} \mathrm{In}$-labeled Isp-PrPOZ(20 kDa). Data are mean \pm SD.

\section{RESULTS}

Isp-PrPOZ-injected group (nonradioactive), ${ }^{90} \mathrm{Y}-\mathrm{Isp}-\mathrm{PrPOZ}$-injected group (0.74 MBq), ${ }^{90} \mathrm{Y}-\mathrm{Isp}-\mathrm{PrPOZ}$-injected group (1.85 MBq), and ${ }^{90}$ Y-Isp-PrPOZ-injected group (3.70 MBq). These solutions (5 $\mu \mathrm{L})$ were intratumorally injected, and tumor size and body weight were measured 2 or 3 times a week. Tumor volumes were calculated by the formula [length $\left.\times(\text { width })^{2}\right] / 2$. From an ethical point of view, mice were sacrificed when the tumor volume reached $750 \mathrm{~mm}^{3}$ or when the body weight dropped below $80 \%$ of the body weight.

Furthermore, apart from the therapeutic studies, we prepared tumorbearing mice ( 5 groups, $n=3$ for each group). The tumor, liver, kidneys, and spleen were excised $7 \mathrm{~d}$ after injection of each preparation, and hematoxylin and eosin staining was conducted for histologic analysis.

\section{Statistical Analysis}

Data are expressed as mean \pm SD or SEM from a minimum of 3 experiments. For multiple comparisons, a 1-way ANOVA with posttest (Tukey-Kramer test) was used. The cumulative probability of survival was estimated in each group with the Kaplan-Meier survival curve analysis, and the results were compared with use of the log-rank test with Bonferroni correction for multiple comparisons. A $P$ value of less than 0.05 was considered a statistically significant difference.

\section{Characterization of ${ }^{111}$ In-Labeled POZ Derivatives}

Thermoresponsive POZ derivatives including EtPOZ, IspPOZ, Isp-PrPOZ, and PrPOZ were prepared according to Figure 2. The physicochemical properties of the synthesized POZ derivatives are characterized as follows. The Tts of EtPOZ, IspPOZ, Isp-PrPOZ (5 kDa), Isp-PrPOZ (10 kDa), Isp-PrPOZ (20 kDa), and PrPOZ were greater than $70^{\circ} \mathrm{C}, 34^{\circ} \mathrm{C}, 26^{\circ} \mathrm{C}, 25^{\circ} \mathrm{C}, 25^{\circ} \mathrm{C}$, and $19^{\circ} \mathrm{C}$, respectively. Solutions of Isp-PrPOZ $\left(\mathrm{Tt}, 25^{\circ} \mathrm{C}\right)$ and $\mathrm{EtPOZ}\left(\mathrm{Tt},>70^{\circ} \mathrm{C}\right)$ were dropped on a hot plate heated to $36.5^{\circ} \mathrm{C}$. Isp-PrPOZ was observed to aggregate within a second, whereas EtPOZ did not aggregate (Supplemental Video 1 [supplemental materials are available at http://jnm.snmjournals.org]). The radiochemical purity of ${ }^{111}$ In-labeled POZ was more than $98 \%$ for all POZ derivatives, and radiochemical yield was $40 \%-70 \%$.

\section{Tumor Retention Analysis}

The intratumoral retention of ${ }^{111}$ Inlabeled POZ derivatives was evaluated after intratumoral injection of probes into $\mathrm{PC}-3$ tumors inoculated in the flanks of BALB/ c-nu/nu mice using a nano syringe. Intratumoral retentions at $1 \mathrm{~d}$ of ${ }^{111} \mathrm{In}$-labeled EtPOZ, IspPOZ, Isp-PrPOZ, and PrPOZ (molecular weight, $\sim 20 \mathrm{kDa}$ ) were $5.6 \%$, $15.8 \%, 73.6 \%$, and $73.9 \%$, respectively (Fig. 3A). Isp-PrPOZ and PrPOZ, with Tts lower than tumor temperature (around $33.5^{\circ} \mathrm{C}$ under isoflurane anesthesia, Fig. 3B), had a significantly higher retention rate than EtPOZ and IspPOZ, which had Tts higher than body temperature. Intratumoral localization of ${ }^{111}$ In-labeled POZ derivatives was evaluated by autoradiography $1 \mathrm{~d}$ after injection (Fig. 3C). EtPOZ and IspPOZ were not retained in the tumor, whereas Isp-PrPOZ and PrPOZ showed localization of radioactivity, suggesting rapid intratumoral self-aggregation. Handling of 
PrPOZ at RT was difficult because of its low $\mathrm{Tt}\left(19^{\circ} \mathrm{C}\right)$; therefore, we used Isp-PrPOZ $\left(\mathrm{Tt}, 25^{\circ} \mathrm{C}\right)$ for further experiments. The high level of retention of radioactivity of ${ }^{111} \mathrm{In}$-Isp-PrPOZ in the tumor was retained 3 and $7 \mathrm{~d}$ after injection $(70.5 \%$ and $69.5 \%$, respectively) (Fig. 3D). A low level of ${ }^{111}$ In-Isp-PrPOZ ( $<5.4$ percentage injected dose at $1 \mathrm{~d}$ after injection) distributed in other normal tissues was observed (Supplemental Table 1).

An in vivo biodistribution study was also performed after intravenous injection of ${ }^{111}$ In-labeled POZ derivatives in normal BALB/c-nu/nu mice (Supplemental Tables 2-5). EtPOZ was cleared relatively rapidly from the blood, with no apparent retention in normal tissues, whereas Isp-PrPOZ and PrPOZ rapidly accumulated in the liver and spleen, in which colloidal compounds usually accumulate, suggesting in vivo self-aggregation of IspPrPOZ and PrPOZ. IspPOZ showed a relatively high accumulation in the kidneys.

Next, the relationship between tumor retention and molecular weight of Isp-PrPOZ was investigated 1 and $7 \mathrm{~d}$ after intratumoral injection of ${ }^{111}$ In-labeled Isp-PrPOZ (5 kDa), Isp-PrPOZ (10 kDa), and Isp-PrPOZ (20 kDa) (Fig. 4A). Tumor retention of radioactivity was $39.4 \%, 54.9 \%$, and $73.0 \% 1 \mathrm{~d}$ after injection, and $14.7 \%$, $45.2 \%$, and $69.5 \% 7 \mathrm{~d}$ after injection for ${ }^{111}$ In-labeled Isp-PrPOZ (5 kDa), Isp-PrPOZ (10 kDa), and Isp-PrPOZ (20 kDa), respectively. Furthermore, retention of radioactivity of Isp-PrPOZ (20 $\mathrm{kDa}$ ) in the tumor $1 \mathrm{~d}$ after injection was independent of the concentration of Isp-PrPOZ, in the range of $100-4,000 \mu \mathrm{M}(68.3 \%$, $71.6 \%, 73.1 \%$, and $62.0 \%$ for $100,500,2,000$, and $4,000 \mu \mathrm{M}$, respectively) (Fig. 4B). There was no significant difference in intratumoral retention rate between the probe concentrations examined. On the basis of these results, we chose $20 \mathrm{kDa}$ and $2,000 \mu \mathrm{M}$ as an effective molecular weight and probe concentration, respectively, for further in vivo therapeutic experiments.

\section{Therapeutic Studies Using ${ }^{90}$ Y-Labeled Isp-PrPOZ}

${ }^{90}$ Y-labeled Isp-PrPOZ was obtained with a radiochemical yield of $31.1 \%$ and radiochemical purity of $96.4 \%$. We studied 5 groups of PC-3 tumor-bearing mice: PBS-injected group (control), nonradioactive yttrium-labeled Isp-PrPOZ-injected group (nonradioactive), ${ }^{90} \mathrm{Y}-\mathrm{Isp}-\mathrm{PrPOZ}-$ injected group (0.74 MBq), ${ }^{90} \mathrm{Y}$-Isp-PrPOZ-injected group (1.85 MBq), and ${ }^{90}$ Y-Isp-PrPOZinjected group (3.70 MBq). ${ }^{90}$ Y-Isp-PrPOZ significantly suppressed tumor growth in a dose-dependent manner compared with PBS and nonradioactive yttrium-Isp-PrPOZ (Figs. 5A and 5B). Because tumor size decreased at $7 \mathrm{~d}$ as well as later time points after injection of ${ }^{90} \mathrm{Y}$-Isp-PrPOZ $(0.74,1.85$, and $3.70 \mathrm{MBq})$, histologic analysis was performed $7 \mathrm{~d}$ after injection of probes. Compared with the control group and nonradioactive yttrium-Isp-PrPOZ group, cytotoxicity, including nuclear fragmentation, was observed in the ${ }^{90} \mathrm{Y}-\mathrm{Isp}-$ PrPOZ-injected groups (Fig. 5C). Cellular damage was most severe in the tumors injected with $3.70 \mathrm{MBq}$ of ${ }^{90} \mathrm{Y}$-Isp-PrPOZ. Survival was prolonged in mice treated with ${ }^{90} \mathrm{Y}$-Isp-PrPOZ compared with the control and nonradioactive yttrium-Isp-PrPOZ-injected groups. Fifty percent of mice survived at $90 \mathrm{~d}$ after injection in the ${ }^{90} \mathrm{Y}$-IspPrPOZ (3.70 MBq)-injected group (Fig. 5D). No obvious loss of body weight or systemic side effects were observed in the ${ }^{90} \mathrm{Y}$-IspPrPOZ-injected group (Fig. 5E). Furthermore, histologic analysis of normal tissues $7 \mathrm{~d}$ after intratumoral injection revealed no in vivo cytotoxicity in the liver, kidneys, and spleen (Fig. 5F).

\section{DISCUSSION}

In this study, we developed novel injectable radiopharmaceuticals for brachytherapy that were water-soluble at RT and quickly selfaggregated in tumor tissue after intratumoral injection by regulating the Tt of POZ. We observed that a solution of Isp-PrPOZ with a Tt of $25^{\circ} \mathrm{C}$ aggregated within a second after placement on a plate heated to body temperature, indicating probable rapid seed formation in tumor tissues after injection into the body. In fact, Isp-PrPOZ was determined to have a 13.1- and 4.7-fold-higher retention rate in the tumor than EtPOZ $\left(>70^{\circ} \mathrm{C}\right)$ and $\operatorname{IspPOZ}\left(34^{\circ} \mathrm{C}\right)$, respectively, $1 \mathrm{~d}$ after injection. Intratumoral temperature of mice under isoflurane anesthesia was around $33.5^{\circ} \mathrm{C}$; thus, IspPOZ $\left(34^{\circ} \mathrm{C}\right)$ actually unexpectedly showed low tumor retention. In vivo biodistribution study by intravenous injection of probes demonstrated that POZ derivatives with Tts lower than body temperature were mainly taken up by the liver and spleen (Supplemental Tables 4 and 5), indicating selfaggregation in circulation.

The intratumoral retention of radioactivity was dependent on POZ molecular weight, but independent of probe concentration. The intratumoral retention of radioactivity improved as molecular weight increased; thus, we hypothesized that a POZ formulation greater than $20 \mathrm{kDa}$ might augment the formation of POZ depots in tumor tissues. A similar injectable radiopharmaceutical, radioiodinated elastinlike polypeptide, has been studied for brachytherapy (6). Liu et al. have demonstrated excellent treatment of tumors with ${ }^{131}$ I-labeled polypeptides, but elastinlike polypeptide had low tumor retention when low concentrations were injected. This characteristic might be problematic because probe concentration is diluted immediately after intratumoral injection. However, the tumor retention of ${ }^{111} \mathrm{In}$ Isp-PrPOZ was retained in the concentration range of $100-4,000 \mu \mathrm{M}$, which allows for the use of a lower dose of the polymer and results in the improvement of biosafety for clinical use. In a previous paper, toxicity studies in rats demonstrated that EtPOZ was safe after intravenous injection, and the maximum tolerated dose was greater than $2 \mathrm{~g} / \mathrm{kg}$ (8). Although we speculated about the low toxicity of Isp-PrPOZ, formal long-term toxicity studies would be required
FIGURE 4. Effect of POZ molecular weight and probe concentration on intratumoral radioactivity retention after injection of ${ }^{111} \mathrm{In}$-Isp-PrPOZ. (A) Radioactivity retention (\%) of ${ }^{111} \mathrm{In}$-Isp-PrPOZ with different molecular weight $(5,10$, and $20 \mathrm{kDa}) 1$ and $7 \mathrm{~d}$ after injection ${ }^{\star \star} P<0.01,{ }^{\star} P<0.05 \mathrm{vs}$ $5 \mathrm{kDa})$. Data are mean \pm SD. (B) Radioactivity retention (\%) of ${ }^{111} \mathrm{In}$-lsp-PrPOZ(20 kDa) with different concentration (100, 500, 2,000, and 4,000 $\mu \mathrm{M}) 1 \mathrm{~d}$ after injection. Data are mean \pm SD.

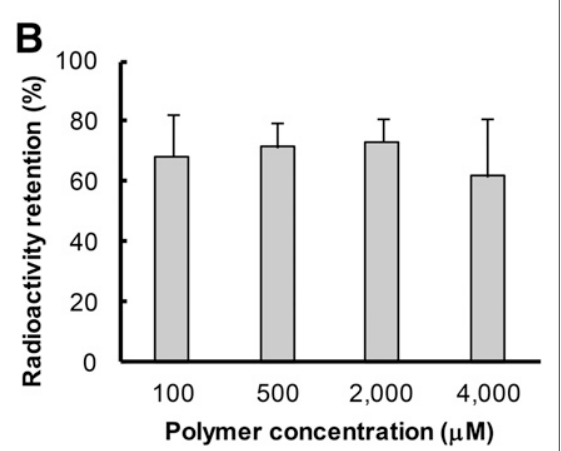

BRACHYTHERAPY With ${ }^{90}$ Y-PolyoXAZOLINE - Sano et al. 


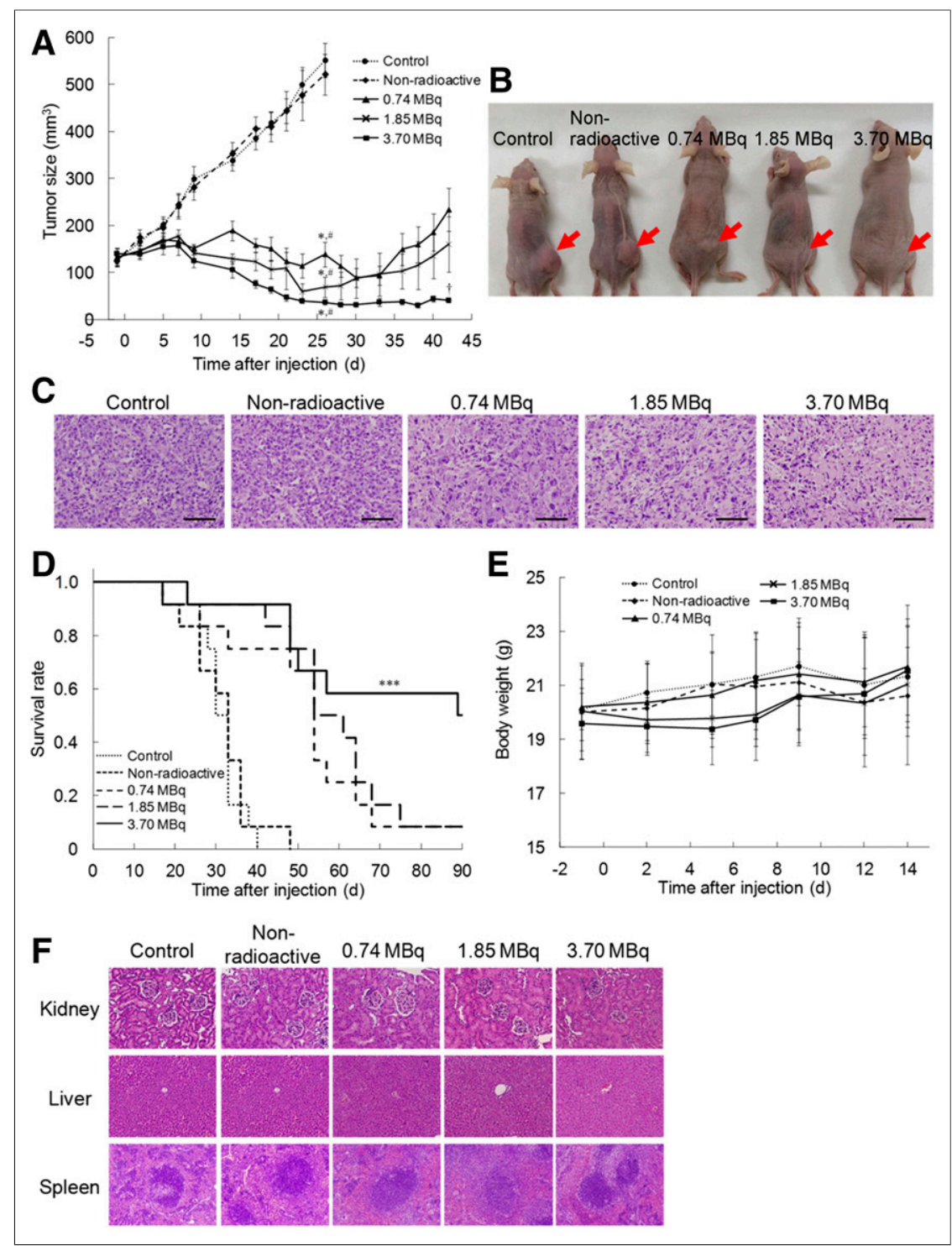

FIGURE 5. Therapeutic effects by intratumoral injection of ${ }^{90}$-labeled Isp-PrPOZ in PC-3 tumorbearing mice. (A) Tumor growth inhibition by ${ }^{90}$ Y-labeled Isp-PrPOZ. Data are mean \pm SEM $(n=9-12$ mice in each group) ( ${ }^{*} P<0.01$ vs. control, ${ }^{\#} P<0.01$ vs. nonradioactive yttrium-Isp-PrPOZ, ${ }^{\dagger} P<0.05$ vs. $0.74 \mathrm{MBq}$ ). (B) Representative pictures of PC-3 tumor-bearing mice $40 \mathrm{~d}$ after treatment with each preparation. (C) Histologic analysis by hematoxylin and eosin staining. Scale bar, $100 \mu \mathrm{m}$. (D) An analysis using Kaplan-Meier survival curve of treatment of ${ }^{90} \mathrm{Y}$-labeled Isp-PrPOZ in PC-3 tumors ( $n=12$ mice in each group) ${ }^{* \star \star} P<0.01$ for treatment compared with other groups.). (E) Change of body weight of tumor-bearing mice treated with each preparation. Data are mean $\pm \operatorname{SD}(n=12)$. (F) Histologic analyses $(n=3)$ of kidney, liver, and spleen at $7 \mathrm{~d}$ after treatment with each preparation.

for clinical translation. Isp-PrPOZ was reasonably localized to a small area $(4 \times 5 \mathrm{~mm}$ as determined from autoradiography data, Fig. 3C), but localization would conversely require a multiple-probe injection regimen for large tumors. By controlling the Tts of POZ used for brachytherapy, a high therapeutic effect for larger tumors via single-probe injection would be expected.

The therapeutic effect of ${ }^{90} \mathrm{Y}$-Isp-PrPOZ was enhanced in a dosedependent manner, with $50 \%$ of mice surviving $90 \mathrm{~d}$ after injection of ${ }^{90}$ Y-Isp-PrPOZ (3.70 MBq). Cytotoxicity such as nuclear fragmentation in the tumor was observed within a week after injection of ${ }^{90}$ Y-Isp-PrPOZ. The injected radioactivity was $5 \%-17 \%$ of radioactivity that was administered in other reports on injectable radiopharmaceuticals for brachytherapy $(5,6)$, leading to decreasing exposure dose for clinical trials. However, there might still be room for improvement in the chemical modification of POZ derivatives. The radiochemical yield of ${ }^{90} \mathrm{Y}$-labeled IspPrPOZ was not high $(\sim 30 \%)$, which might be improved by changing the chelator from DOTA to diethylenetriamine pentaacetic acid, the clinical chelator for ${ }^{90}$ Y-labeled ibritumomab tiuxetan (17).

Although this therapeutic study was conducted using the PC-3 (human prostate cancer)-bearing mouse model, we hypothesized that this probe could be applied to other cancers including breast, brain, and gynecologic that are currently treated by capsule seed-type brachytherapy (18-21). Brachytherapy is also conducted to prevent tumor recurrence after surgical treatment. Recently, breast-conserving surgery with whole-breast radiation therapy has become the standard treatment of early-stage breast cancer (22). In these cases, injectable radiopharmaceuticals, which do not require seed removal and have decreased difficulty of handling, would be extremely useful.

\section{CONCLUSION}

We demonstrated the potential of the novel injectable radiopharmaceutical ${ }^{90} \mathrm{Y}$ labeled POZ for brachytherapy. The $\mathrm{Tt}$ of Isp-PrPOZ was determined to be $25^{\circ} \mathrm{C}$, that is, Isp-PrPOZ is soluble at RT and selfaggregates at body temperature. The optimized ${ }^{111}$ In-Isp-PrPOZ (20 kDa, 2,000 $\left.\mu \mathrm{M}\right)$ was highly retained $7 \mathrm{~d}$ after injection, and ${ }^{90}$ Y-Isp-PrPOZ exhibited a high therapeutic effect via intratumoral aggregation with no damage to normal tissue. Therefore, ${ }^{90} \mathrm{Y}$-IspPrPOZ would be used for treating cancer with currently marketed capsule-type seeds.

\section{DISCLOSURE}

This research was supported by JSPS KAKENHI grants 16H06256 and 26860991 (Kohei Sano), Shimazu Science Foundation (Kohei Sano), and Takeda Science Foundation (Kohei Sano). This work was partly supported by the Innovative Techno-Hub for Integrated Medical Bio-imaging Project of the Special Coordination Funds for Promoting Science and Technology, from the Ministry of Education, Culture, Sports, Science and Technology (MEXT), Japan. No other potential conflict of interest relevant to this article was reported.

\section{REFERENCES}

1. Tanderup K, Ménard C, Polgar C, Lindegaard JC, Kirisits C, Pötter R. Advancements in brachytherapy. Adv Drug Deliv Rev. 2017;109:15-25.

2. Perera M, Krishnananthan N, Lindner U, Lawrentschuk N. An update on focal therapy for prostate cancer. Nat Rev Urol. 2016;13:641-653. 
3. Older RA, Synder B, Krupski TL, Glembocki DJ, Gillenwater JY. Radioactive implant migration in patients treated for localized prostate cancer with interstitial brachytherapy. J Urol. 2001;165:1590-1592.

4. Hrubý M, Subr V, Kucka J, Kozempel J, Lebeda O, Sikora A. Thermoresponsive polymers as promising new materials for local radiotherapy. Appl Radiat Isot. 2005;63:423-431.

5. Hruby M, Pouckova P, Zadinova M, Kucka J, Lebeda O. Thermoresponsive polymeric radionuclide delivery system: an injectable brachytherapy. Eur J Pharm Sci. 2011;42:484-488.

6. Liu W, McDaniel J, Li X, et al. Brachytherapy using injectable seeds that are self-assembled from genetically encoded polypeptides in situ. Cancer Res. 2012;72:5956-5965.

7. de la Puente P, Azab AK. Delivery systems for brachytherapy. J Control Release. 2014;192:19-28.

8. Viegas TX, Bentley MD, Harris JM, et al. Polyoxazoline, chemistry, properties, and application in drug delivery. Bioconjug Chem. 2011;22:976-986.

9. Hruby M, Filippov SK, Panek J, et al. Polyoxazoline thermoresponsive micelles as radionuclide delivery systems. Macromol Biosci. 2010;10:916-924

10. Adams N, Schubert US. Poly(2-oxazolines) in biological and biomedical application contexts. Adv Drug Deliv Rev. 2007;59:1504-1520.

11. Kanazaki K, Sano K, Makino A, Homma T, Ono M, Saji H. Polyoxazoline multivalently conjugated with indocyanine green for sensitive in vivo photoacoustic imaging of tumors. Sci Rep. 2016;6:33798.

12. Luxenhofer R, Han Y, Schulz A, et al. Poly(2-oxazoline)s as polymer therapeutics. Macromol Rapid Commun. 2012;33:1613-1631.

13. Roy D, Brooks WL, Sumerlin BS. New directions in thermoresponsive polymers. Chem Soc Rev. 2013;42:7214-7243.

14. Koukouraki S, Strauss LG, Georgoulias V, et al. Evaluation of the pharmacokinetics of ${ }^{68} \mathrm{Ga}$-DOTATOC in patients with metastatic neuroendocrine tumors scheduled for ${ }^{90}$ Y-DOTATOC therapy. Eur J Nucl Med Mol Imaging. 2006; 33:460-466.

15. Gaertner FC, Luxenhofer R, Blechert B, Jordan R, Essler M. Synthesis, biodistribution and excretion of radiolabeled poly(2-alkyl-2-oxazoline)s. J Control Release. 2007;119:291-300.

16. Huang H, Hoogenboom R, Leenen MA, et al. Solvent-induced morphological transition in core-cross-linked block copolymer micelles. J Am Chem Soc. 2006;128:3784-3788.

17. Wagner HN Jr, Wiseman GA, Marcus CS, et al. Administration guidelines for radioimmunotherapy of non-Hodgkin's lymphoma with ${ }^{90}$ Y-labeled anti-CD20 monoclonal antibody. J Nucl Med. 2002;43:267-272.

18. Keller BM, Pignol JP, Rakovitch E, Sankreacha R, O’Brien P. A radiation badge survey for family members living with patients treated with a ${ }^{103} \mathrm{Pd}$ permanent breast seed implant. Int J Radiat Oncol Biol Phys. 2012;70:13741380 .

19. Petr MJ, McPherson CM, Breneman JC, Warnick RE. Management of newly diagnosed single brain metastasis with surgical resection and permanent I-125 seeds without upfront whole brain radiotherapy. J Neurooncol. 2009;92:393400 .

20. Keys HM, Roberts JA, Brunetto VL, et al. A phase III trial of surgery with or without adjunctive external pelvic radiation therapy in intermediate risk endometrial adenocarcinoma: a Gynecologic Oncology Group study. Gynecol Oncol. 2004;92:744-751.

21. Ashamalla H, Rafla S, Zaki B, Ikoro NC, Ross P. Radioactive gold grain implants in recurrent and locally advanced head-and-neck cancers. Brachytherapy. 2002;1:161-166.

22. Veronesi U, Cascinelli N, Mariani L, et al. Twenty-year follow-up of a randomized study comparing breast-conserving surgery with radical mastectomy for early breast cancer. N Engl J Med. 2002;347:1227-1232. 